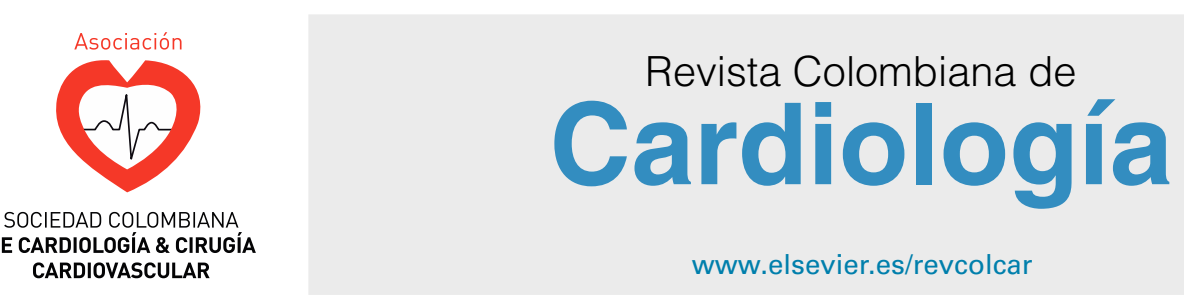

CARDIOLOGÍA DEL ADULTO - ARTÍCULO ORIGINAL

\title{
Modelo de costos asociados al ataque cerebrovascular y los eventos adversos en pacientes con fibrilación auricular no valvular tratados con warfarina
}

\author{
José A. Mendoza-Sánchez*, Federico A. Silva, Lady M. Rangel-Celis, \\ Jaime E. Arias y Eduardo A. Zuñiga-Sierra
}

Grupo de Ciencias Neurovasculares Fundación Cardiovascular de Colombia, Floridablanca, Colombia

Recibido el 13 de julio de 2017; aceptado el 3 de diciembre de 2018

Disponible en Internet el 10 de abril de 2019

\author{
PALABRAS CLAVE \\ Accidente \\ cerebrovascular; \\ Eventos adversos; \\ Costos; \\ Warfarina
}

\begin{abstract}
Resumen
Objetivo: estimar el número y el costo médico directo del accidente cerebrovascular y los eventos adversos en usuarios de warfarina por fibrilación auricular.

Métodos: se utilizó la metodología del análisis de impacto presupuestario para estimar el número de casos y el costo de los eventos. Se calculó la población diana con base en información poblacional de Colombia, así como las tasas de uso de warfarina según el riesgo y la distribución del tiempo en rango terapéutico de la misma. Se estimaron los eventos con base en probabilidades reportadas, y los costos utilizados en el modelo fueron calculados con información de una cohorte de pacientes con accidente cerebrovascular que se lleva a cabo en la Fundación Cardiovascular de Colombia.

Resultados: la población diana fue de 84623 pacientes en 1 año. El costo total de los accidentes cerebrovasculares y los sangrados fue de $\$ 36852236476$ millones COP. El costo total de los accidentes cerebrovasculares fue $\$ 5004588241$ COP; el costo total de las hemorragias intracraneales fue $\$ 11875730234$ COP y el de las hemorragias gastrointestinales fue $\$ 19246$ 023614 COP. El sangrado retroperitoneal, la epistaxis y el sangrado del tracto urinario fueron $\$ 735894387$ COP. El análisis de sensibilidad mostró que un ajuste en $10 \%$ de los tiempos en rango terapéutico podría ahorrar \$4 211957037 COP.

Conclusiones: el costo del accidente cerebrovascular y de los eventos adversos por warfarina es grande. Sin embargo, un ajuste de los tiempos en rango terapéutico podría ahorrar costos, lo que significa vidas de pacientes.

(c) 2019 Sociedad Colombiana de Cardiología y Cirugía Cardiovascular. Publicado por Elsevier España, S.L.U. Este es un artículo Open Access bajo la licencia CC BY-NC-ND (http:// creativecommons.org/licenses/by-nc-nd/4.0/).
\end{abstract}

\footnotetext{
* Autor para correspondencia.

Correo electrónico: jmendoza45@msn.com (J.A. Mendoza-Sánchez).
} 


\section{KEYWORDS}

Cerebrovascular

accident;

Stroke;

Adverse events;

Costs;

Warfarin

\section{Model of costs associated with stroke and adverse events in patients with non-valvular atrial fibrillation treated with warfarin}

\begin{abstract}
Objective: The aim of this study is to estimate the number and direct medical cost of cerebrovascular accident (stroke) and the adverse events in patients on warfarin due to atrial fibrillation.

Methods: A budget impact analysis methodology was used to estimate the number of cases and cost of the events. The target population was based on Colombian population information, as well as the rates of use of warfarin according to risk and the distribution of its therapeutic time range. The events were estimated base on reported probabilities, and the costs used in the model were calculated from a cohort of stroke patients that was performed in the Cardiovascular Foundation of Colombia.

Results: The target population consisted of 84,623 patients in 1 year. The total cost the strokes and bleeding was $€ 10,426,275.28$ (\$36 852236476 million COP (Note: 3,500/3,000Colombian Pesos (COP) $=1$ Euro approx). The total cost strokes was $€ 1,415,903.61$ ( $\$ 5004588241$ COP). The total costs of intracranial and gastrointestinal bleeding was $€ 3,359,894.66$ ( $\$ 11875730$ 234 COP) and $€ 5,445,106.17$ ( $\$ 19246023614$ COP), respectively. Retroperitoneal bleeding, epistaxis (nosebleeds) and urinary tract bleeding was $€ 208,200.05$ (\$735 894387 COP). The sensitivity analysis showed that an adjustment of $10 \%$ in the therapeutic time ranges could save $€ 1,191,651.52$ (\$4 211957037 COP).

Conclusions: The cost of the cerebrovascular accident and the adverse effects due to warfarin is large. However, an adjustment in the therapeutic range times could save costs, which means patient lives.

(c) 2019 Sociedad Colombiana de Cardiología y Cirugía Cardiovascular. Published by Elsevier España, S.L.U. This is an open access article under the CC BY-NC-ND license (http:// creativecommons.org/licenses/by-nc-nd/4.0/).
\end{abstract}

\section{Introducción}

La fibrilación auricular es un trastorno crónico que afecta cerca de 2.3 millones de personas en Estados Unidos y 4.5 millones de personas en Europa ${ }^{1,2}$, aumenta 4 veces el riesgo de accidente cerebrovascular y causa el $15 \%$ de los accidentes cerebrovasculares isquémicos de origen cardioembólico ${ }^{3}$. La warfarina es uno de los anticoagulantes orales recomendados para la prevención del accidente cerebrovascular en pacientes con fibrilación auricular ${ }^{4}$ y continúa siendo de primera línea en Colombia; sin embargo, es reconocido como uno de los principales medicamentos implicados en reacciones medicamentosas adversas ${ }^{5}$, las cuales en su mayoría son hemorragias, que van desde sangrados menores (epistaxis, sangrado genito-urinario y sangrado retroperitoneal), hasta sangrados más graves (sangrado gastrointestinal y hemorragia intracraneal ${ }^{6}$. La prevalencia de eventos medicamentosos adversos por warfarina puede llegar a ser $6.5 \%$, de los cuales el $80 \%$ requieren hospitalización, con una mortalidad global de $0.15 \%$.

El costo del accidente cerebrovascular es enorme, en Europa se estima que cuesta $€ 64$ billones cada año y en los Estados Unidos cuesta $\$ 53.9$ billones de dólares, por eso la prevención del accidente cerebrovascular por AF es de alta prioridad para los tomadores de decisiones en salud ${ }^{8}$. En Colombia la información sobre costos directos del accidente cerebrovascular es escasa; sin embargo, con base en información de costos utilizados en estudios económicos publicados se estimó que los costos totales (costos médicos directos e indirectos) para el año 2008 podrían ser de $\$ 450$ mil millones de pesos colombianos (COP) ${ }^{9}$. Pero, se desconoce el costo para el sistema de salud de los eventos clínicos (accidentes cerebrovasculares y hemorragias) relacionados con el uso de warfarina por indicación de fibrilación auricular, pese a que el costo médico anual aumenta con las hemorragias intracraneales y gastrointestinales en un $64 \%$ y $49 \%$ respectivamente por paciente ${ }^{4}$. Se sabe que el riesgo de accidente cerebrovascular en fibrilación auricular disminuye con el uso de warfarina según resultados de ensayos clínicos controlados y estudios en práctica clínica (64 y 27\%) respectivamente ${ }^{10}$. También se sabe que las hemorragias disminuyen la adherencia, hecho que impacta en la tasa de uso del medicamento en la población y se debe tener en cuenta al modelar en anticoagulación oral ${ }^{8,11}$.

Con el fin de maximizar los beneficios y minimizar riesgos del uso de la warfarina, se recomienda ajustar las dosis de warfarina (INR 2-3); sin embargo, el mantenimiento del paciente en rango terapéutico de warfarina no asegura la no aparición de un accidente cerebrovascular o hemorragia ${ }^{12-14}$; también, las hemorragias intracraneales por warfarina se relacionan con mayor severidad, mortalidad y costo en comparación con pacientes no usuarios de warfarina ${ }^{15,16}$. Incluir la mayor parte de las variables relacionadas en anticoagulación oral con warfarina es fundamental para mejorar las estimaciones de los modelos económicos en farmacoeconomía, que son importantes para la toma de decisiones. El objetivo de este estudio está dirigido a estimar el costo médico directo en un año de los 


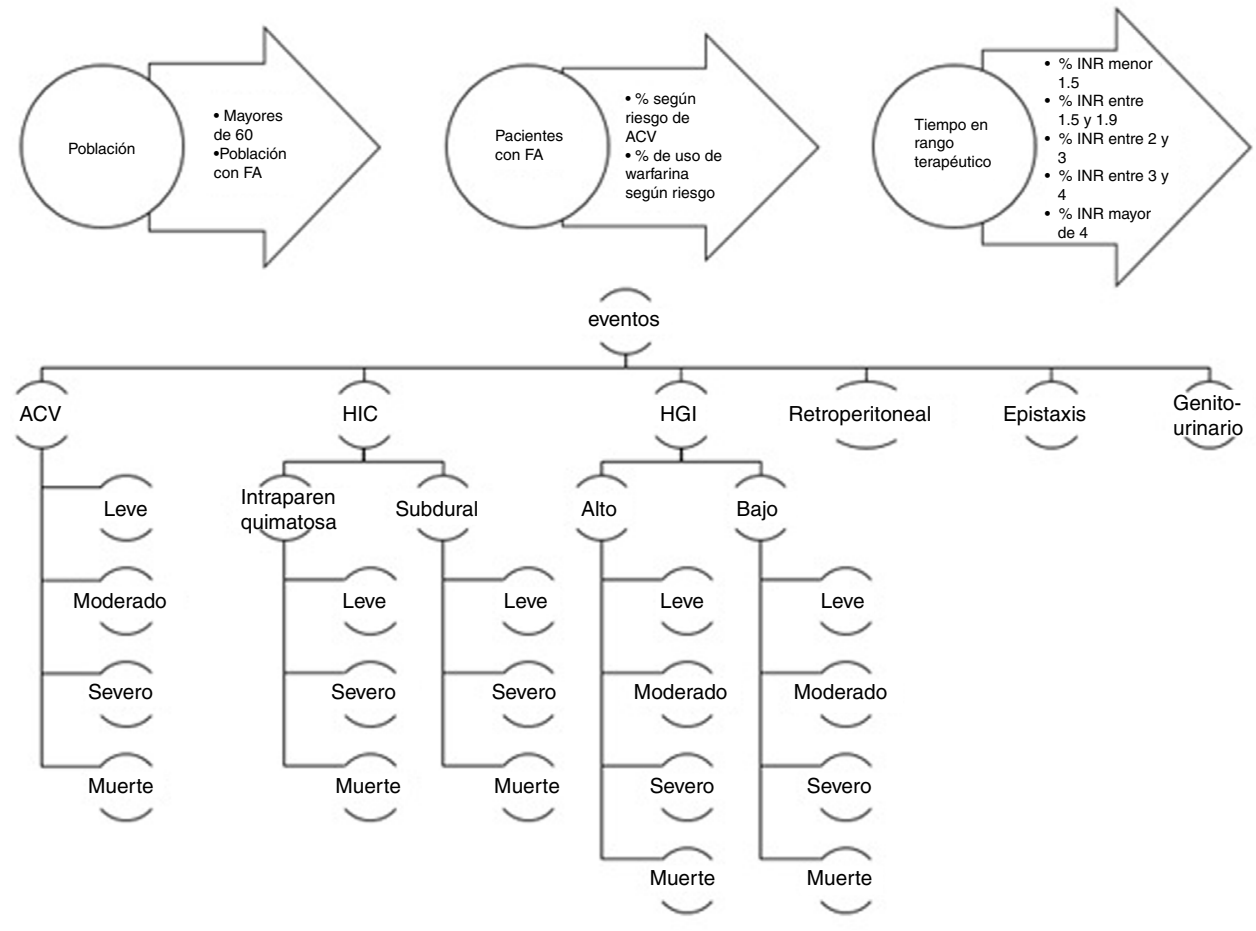

Figura 1 Esquema del modelo en anticoagulación oral.

Descripción del modelo utilizado para el cálculo de los eventos.

eventos relacionados con la anticoagulación con warfarina por fibrilación auricular, teniendo en cuenta parámetros para el modelo como la tasa de uso del medicamento según el riesgo de accidente cerebrovascular, el porcentaje de tiempo en rango terapéutico y la localización de las hemorragias intracraneales y gastrointestinales a través del empleo de la metodología del análisis de impacto presupuestario para este estudio.

\section{Métodos}

El modelo utilizado en este estudio fue diseñado para calcular el número de eventos y el costo en un año en población colombiana. Se utilizó la metodología del análisis de impacto presupuestario para este estudio ${ }^{17,18}$. Se estimó el número de pacientes con fibrilación auricular y usuarios de warfarina en Colombia. El cálculo se basó en datos locales publicados de prevalencia de fibrilación auricular en población mayor de 60 años, que fue una estimación de registros de historias clínicas de un hospital ${ }^{19}$. El cálculo de la población mayor de 60 años con fibrilación auricular (población diana) fue clasificado según el riesgo en: bajo, medio y alto de accidente cerebrovascular $\left(\mathrm{CHADS}_{2}\right)^{20}$. Se tomaron las tasas de utilización de warfarina según el riesgo de accidente cerebrovascular, con lo que se estimó el número de usuarios de warfarina según el riesgo (población diana). Posterior al cálculo de la población usuaria de warfarina, se estimó el número de eventos multiplicando la probabilidad de cada evento según el tiempo en rango terapéutico, por la población diana. Las probabilidades de acuerdo con el tiempo en rango terapéutico (TRT) y la severidad fueron extraídos de los estudios de Hylek et al., Rosand et al. Chen et al. y Leigh et al. ${ }^{12,21-23}$. La figura 1, muestra el esquema del modelo utilizado en este estudio, que es el mismo que se emplea para los estudios de impacto presupuestario en farmacoeconomía con horizonte temporal de un año. Se estimaron los costos de cada evento según la severidad y la localización (hemorragia intracraneal intra-parenquimatosa, subdural y gastrointestinal alta y baja). Se utilizó un costeo tipo "de abajo a arriba" de los datos de una cohorte de pacientes con enfermedad cerebrovascular que se lleva a cabo en la Fundación Cardiovascular de Colombia. Los grandes supuestos de este modelo son los siguientes: todos los pacientes tenían igual riesgo de base; se asumió que las probabilidades de los eventos fueron similares a las de la cohorte de Hylet et al., en la cual la edad media fue 72 años y el porcentaje de mujeres fue $43 \%$; los porcentajes de factores de riesgo mayores para accidente cerebrovascular fueron 9,3\% para accidente cerebrovascular previo, 51\% para hipertensión y $30,8 \%$ para falla cardiaca; el modelo también asumió diferentes tiempos de rango terapéutico (INR) y sus probabilidades según la severidad.

El modelo para el cálculo de los eventos tuvo en cuenta los siguientes componentes:

1. Población total: para el caso la población colombiana, según datos del DANE ${ }^{24}$.

2. Población enferma (diagnóstico de fibrilación auricular) estimación de prevalencia ${ }^{19}$.

3. Población objetivo: pacientes con fibrilación auricular usuarios de warfarina ${ }^{20}$.

4. Cálculo de los eventos; probabilidades ${ }^{12,21-23}$.

5. Utilización de recursos; cálculo de costos por severidad y localización. 
A continuación se da una explicación más detallada de los componentes del modelo:

- Población "blanco" o potenciales usuarios: para la estimación de la población en riesgo de evento adverso por anticoagulación oral en fibrilación auricular no valvular, se utilizó la información del DANE de la población colombiana correspondiente al año 2016, con los datos reportados de pacientes mayores de 60 años y una prevalencia de $3,6 \%$ de fibrilación auricular en pacientes mayores de 60 años según estudio publicado por Roselli et al. en el que se estimó la prevalencia de fibrilación auricular en Colombia en los pacientes mayores de 60 años con diagnóstico de fibrilación auricular. Por datos de estudios fármaco-epidemiológicos, las tasas de uso del medicamento reportadas en el estudio de Mohammed et al. ${ }^{20}$, se calcularon con base en los usuarios de warfarina según el riesgo de accidente cerebrovascular (leve, moderado y severo), las cuales fueron de $37 ; 47,15$ y $54,8 \%$ respectivamente.

- Calculo del número de eventos: con la información estimada de la población usuaria de warfarina por fibrilación auricular no valvular en Colombia, se calculó el número de eventos para cada una de las variables incluidas en nuestro modelo. Se calcularon los siguientes eventos: accidente cerebrovascular, hemorragia intracraneal, hemorragia gastrointestinal, epistaxis, y sangrados urinario y retroperitoneal.

- Estimación de costos: se obtuvieron costos para el modelo con base en información de una cohorte de pacientes con accidente cerebrovascular de la Fundación Cardiovascular de Colombia; la opinión de expertos permitió la estimación de tiempos promedio de hospitalización, solicitud de exámenes, días de estancia en unidad de cuidados intensivos, indicaciones quirúrgicas por severidad (craneotomía, endoscopia de vías digestivas, colonoscopia, colostomía, gastrostomía y taponamiento nasal posterior) sin considerar tratamiento quirúrgico para el sangrado retroperitoneal.

\section{Resultados}

La estimación de la población diana (pacientes mayores de 60 años con diagnóstico de fibrilación auricular tratados con warfarina) fue de 84623 pacientes en 1 año. El número total de eventos fue de 6583 casos con costo total en un año de 36852236476 millones COP, costo promedio por paciente de \$5 598091 COP y probabilidad para cualquier evento de $0,78 \%$ (tabla 3,). Para el grupo de accidente cerebrovascular el mayor número de casos fueron leves (493),

Tabla 1 Parámetros del modelo

\begin{tabular}{|c|c|c|c|c|c|}
\hline Parámetros & & Parámetros & & Parámetros & \\
\hline $\begin{array}{l}\text { Tiempo en rango } \\
\text { terapéutico }\end{array}$ & & $\begin{array}{l}\text { Severidad accidente } \\
\text { cerebrovascular }\end{array}$ & & Localización HIC & \\
\hline INR menor 1.5 & $6 \%(38)$ & INR $<2$ & & Intracerebral & $0.42(39)$ \\
\hline INR entre 1.5 y 1.9 & $19 \%(38)$ & Leve & $0.41(12)$ & Subdural & $0.3(39)$ \\
\hline INR entre 2 y 3 & $59 \%(38)$ & Moderado & $0.44(12)$ & Severidad HIC & \\
\hline INR entre 3.1 y 3.9 & $13 \%(38)$ & Severo & $0.06(12)$ & Leve & $0.17(23)$ \\
\hline INR mayor de 4 & $4 \%(38)$ & Fatal & $0.09(12)$ & Severo & $0.56(23)$ \\
\hline $\begin{array}{l}\text { Clasificación de riesgo de } \\
\text { accidente cerebrovascular }\end{array}$ & & INR > 2 & Fatal & $0.27(23)$ & \\
\hline Riesgo bajo & $24 \%(20)$ & Leve & $0.57(12)$ & Probabilidad HGI & \\
\hline Riesgo moderado & $35 \%(20)$ & Moderado & $0.38(12)$ & Prob HGI INR 1-1.4 & $0.021(22)$ \\
\hline Riesgo alto & $41 \%(20)$ & Severo & $0.04(12)$ & Prob HGI INR 1.5-1.9 & $0.039(22)$ \\
\hline $\begin{array}{l}\text { Uso de warfarina por grupos } \\
\text { de riesgo }\end{array}$ & Fatal & $0.01(12)$ & Prob HGI INR 2-3 & $0.045(22)$ & \\
\hline Riesgo bajo & $37 \%(20)$ & Probabilidad HIC & & Prob HGI INR > 3 & $0.143(22)$ \\
\hline Riesgo medio & $47.15 \%(20)$ & Prob HIC INR 1-1.4 & $0.005(12)$ & Localización HGI & \\
\hline Riesgo bajo & $54.88 \%(20)$ & Prob HIC INR 1.5-1.9 & $0.003(12)$ & Alto & $0.7(22)$ \\
\hline $\begin{array}{l}\text { Probabilidades accidente } \\
\text { cerebrovascular }\end{array}$ & & Prob HIC INR 2-3 & $0.00363(12)$ & Bajo & $0.3(22)$ \\
\hline $\begin{array}{l}\text { Prob accidente } \\
\text { cerebrovascular INR 1-1.4 }\end{array}$ & $0.077(12)$ & Prob HIC INR 3.1-3.9 & $0.006(12)$ & Severidad HGI & \\
\hline $\begin{array}{l}\text { Prob accidente } \\
\text { cerebrovascular INR 1.5-1.9 }\end{array}$ & $0.019(12)$ & Prob HIC INR > 4 & $0.05683(12)$ & Leve & $0.44(23)$ \\
\hline $\begin{array}{l}\text { Prob accidente } \\
\text { cerebrovascular INR 2-3 }\end{array}$ & $0.0055(12)$ & Otros sangrados & & Moderado & $0.38(23)$ \\
\hline $\begin{array}{l}\text { Prob accidente } \\
\text { cerebrovascular INR 3.1-3.9 }\end{array}$ & $0.0065(12)$ & Genitourinario & $0.00535(23)$ & Severo & $0.09(23)$ \\
\hline \multirow{2}{*}{$\begin{array}{l}\text { Prob accidente } \\
\text { cerebrovascular INR }>4\end{array}$} & $0.019(12)$ & Epistaxis & $0.00107(23)$ & Fatal & $0.09(23)$ \\
\hline & & Retroperitoneal & $0.00149(23)$ & & \\
\hline
\end{tabular}


Tabla 2 Costos utilizados

\begin{tabular}{|c|c|c|c|}
\hline Eventos & Costos & Eventos & Costos \\
\hline Accidente cerebrovascular & & HIG & \\
\hline Accidente cerebrovascular leve & $\$ 2960345$ & HGI alta leve & $\$ 2287354$ \\
\hline Accidente cerebrovascular moderado & $\$ 3235284$ & HGI alta moderado & $\$ 3352181$ \\
\hline Accidente cerebrovascular severo & $\$ 25647404$ & HGI alta severa & $\$ 5481835$ \\
\hline HIC & & HIG & \\
\hline HIC leve & $\$ 6462287$ & HGI baja leve & $\$ 3517081$ \\
\hline HIC moderada & $\$ 30921966$ & HGI baja moderada & $\$ 5646735$ \\
\hline HIC severa & $\$ 50902604$ & HGI baja severa & $\$ 12355145$ \\
\hline \multicolumn{4}{|l|}{ OTROS } \\
\hline Sangrado retroperitoneal & $\$ 800000$ & Sangrado genitourinario & $\$ 1000000$ \\
\hline Epistaxis & $\$ 1200000$ & & \\
\hline
\end{tabular}

HIC: hemorragia intracraneal, HGI: hemorragia gastrointestinal.

Tabla 3 Número y costos de los eventos

\begin{tabular}{|c|c|c|}
\hline Eventos & \# eventos & Costos \\
\hline Accidente cerebrovascular & casos & Costo total \\
\hline Leve & 492.71 & $\$ 1458601533$ \\
\hline Moderado & 444.55 & $\$ 1438246029$ \\
\hline Severo & 56.33 & $\$ 1444592305$ \\
\hline Muerte & 66.31 & $\$ 663148374$ \\
\hline Total & 1059.90 & $\$ 5004588241$ \\
\hline \multicolumn{3}{|l|}{$\begin{array}{l}\text { Hemorragia intracraneal } \\
\text { Intraparenquimatosa }\end{array}$} \\
\hline Leve & 36.43 & $\$ 235390805$ \\
\hline Severa & 119.99 & $\$ 6107765542$ \\
\hline Muerte & 57.85 & $\$ 578519623$ \\
\hline Total & 214.27 & $\$ 6921675970$ \\
\hline \multicolumn{3}{|l|}{ Subdural } \\
\hline Leve & 26.02 & $\$ 168136289$ \\
\hline Severa & 85.71 & $\$ 4362689673$ \\
\hline Muerte & 41.32 & $\$ 413228302$ \\
\hline Total & 153.05 & $\$ 4944054264$ \\
\hline \multicolumn{3}{|l|}{$\begin{array}{l}\text { Hemorragia } \\
\text { gastrointestinal alta }\end{array}$} \\
\hline Leve & 1364 & $\$ 3120941193$ \\
\hline Moderado & 1201 & $\$ 4026368212$ \\
\hline Severo & 281 & $\$ 1542120811$ \\
\hline Muerte & 284 & $\$ 2844755471$ \\
\hline Total & 3131.34 & $\$ 11534185688$ \\
\hline \multicolumn{3}{|l|}{$\begin{array}{l}\text { Hemorragia } \\
\text { gastrointestinal baja }\end{array}$} \\
\hline Leve & 596.04 & $\$ 2096335040$ \\
\hline Moderado & 514.77 & $\$ 2906743099$ \\
\hline Severo & 120.56 & $\$ 1489578871$ \\
\hline Muerte & 121.92 & $\$ 1219180916$ \\
\hline Total & 1353.29 & \$7 711837926 \\
\hline Retroperitoneal & 126.92 & $\$ 101538799$ \\
\hline Epistaxis & 453.08 & $\$ 543695946$ \\
\hline Tracto urinario & 90.66 & $\$ 90659642$ \\
\hline TOTAL & 6583 & $\$ 36852236476$ \\
\hline $\begin{array}{l}\text { Costo promedio por } \\
\text { paciente }\end{array}$ & & $\$ 5598091$ \\
\hline
\end{tabular}

seguidos de moderados (445), muerte (65) y severos (55). El total de casos de accidente cerebrovascular fue 1060 , costo total de \$5 004588241 COP; la menor cantidad de casos fueron severos pero el costo fue similar a los leves y moderados. Las hemorragias intracraneales en total fueron 367 ; intraparenquimatosa 214 , subdurales 153 . El costo total de las hemorragias intracraneales fue \$11 875730234 COP. La mayor cantidad de casos se trató de severas en los dos tipos: 119 y 85 respectivamente. El costo de acuerdo con la localización de la hemorragia cerebral fue \$6921 675970 COP para la intraparenquimatosa, y 4944054264 COP para la subdural.

El número estimado de hemorragias gastrointestinales fue de 4485 casos; 3131 gastrointestinal alto y 1353 gastrointestinal bajo, con un costo de \$19 246023214 COP. En los dos grupos la mayor cantidad de casos correspondió a leves seguido de moderado, severo y muerte. El costo por localización fue: \$11534185688 COP para gastrointestinal alto y $\$ 7711837926$ COP gastrointestinal bajo. El sangrado retroperitoneal, la epistaxis y el sangrado de tracto genitourinario correspondió a 126, 453 y 90 casos respectivamente. El costo de cada uno de ellos fue de \$101 538799 COP, \$543 695946 COP y $\$ 90659642$ COP respectivamente.

\section{Análisis de sensibilidad}

Se realizó un análisis de sensibilidad determinístico tipo tornado (fig. 2). Las variables que más influenciaron el modelo se presentan de mayor a menor sensibilidad al cambio (del costo total) en orden descendente. Todos los parámetros del modelo fueron incluidos en el análisis de sensibilidad; se definió un rango de prueba del $-10 \%$ a $10 \%$, utilizando valores de la mediana del caso base de las variables. Las cinco variables que más afectan el modelo fueron tiempo en rango terapéutico (TRT) entre 2 y 3 , costo hemorragia intracraneal (HIC) severa, probabilidad de hemorragia gastrointestinal en INR en rango terapéutico, TRT entre 3 y 4 , y probabilidad de hemorragia gastrointestinal en INR mayor de 3 . El tiempo en rango terapéutico entre 2 y 3 puede afectar el modelo en un $29,78 \%$, con variación de $\$ 2935706046$. EL costo de la HIC severa puede modificar el modelo en un $15 \%$, con variación de \$2 094091 043. La probabilidad de hemorragia gastrointestinal en rango de INR entre 2 y 3 puede afectar el modelo 


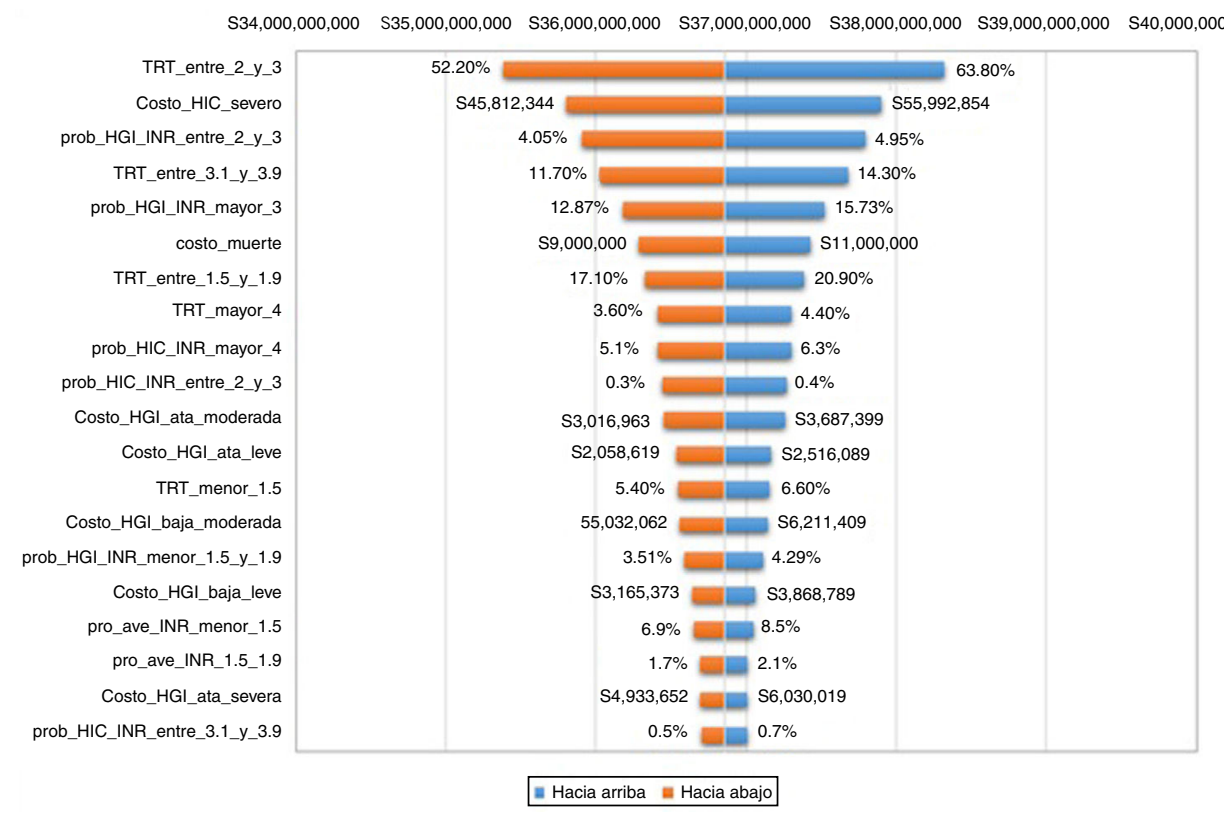

Figura 2 Análisis de sensibilidad tipo tornado.

Análisis univariado de los parámetros que más influyen en el modelo en orden ascendente a descendente, las variaciones en los parámetros de base $\pm 10 \%$ y su influencia en el costo total. Rangos de variación: TRT_entre_3_y_4: \$1 652 260 741, TRT_entre_1.5_y_1.9: \$1 058675 257, TRT_mayor_4: \$894 840 526; TRT_menor_1.5: \$606 180514.

en un 12.33\%, variación de $\$ 1888656284$. El TRT entre 3 y 4 puede afectar el modelo en $9.43 \%$ con rango $\$ 1652$ 260741 , y la probabilidad de hemorragia gastrointestinal en INR mayor de 3 puede afectar el modelo $6.25 \%$, con rango $\$ 1345215338$. El rango de costo de los tiempos fuera de rango terapéutico de warfarina es de \$4 211957037 COP.

\section{Discusión}

Este modelo fármaco-económico estimó el costo de los eventos adversos y accidente cerebrovascular en población colombiana usuaria de warfarina por indicación de fibrilación auricular no valvular. La mayor parte de los costos se debe al manejo de las hemorragias gastrointestinales e intracraneales (52 y $32 \%$ de los costos, respectivamente) y en menor medida al costo del accidente cerebrovascular y de las otras hemorragias. El mayor costo de las hemorragias obedece al número total de hemorragias y es mayor al número de accidente cerebrovascular, mientras que el costo del manejo de las hemorragias que no son leves puede ser mayor al costo de un accidente cerebrovascular, que puede explicarse por el costo de las intervenciones médicas diagnósticas en la hemorragia gastrointestinal (endoscopia y colonoscopia) o en hemorragia intracraneal (tratamiento quirúrgico) por ejemplo. El menor costo del total del accidente cerebrovascular también significa que se cumple en parte con el objetivo de la terapia anticoagulante en la prevención del mismo, pese a que en este modelo se utilizó como parámetro un $59 \%$ de los pacientes en rango terapéutico (INR 2-3), 19\% entre 1,5 y 1,9 y $13 \%$ entre 3,1 y 3,9 de INR; tiempos de rango terapéutico utilizados en este estudio menores a los reportados en ensayos clínicos controlados ${ }^{25}$.
El análisis de sensibilidad tipo tornado identificó los parámetros que más influenciaron el costo total. Por su parte, los parámetros que más influenciaron el modelo fueron aquellos relacionados con el porcentaje de tiempo en rango terapéutico (modificables), costos y probabilidades (no modificables). El tiempo en rango terapéutico entre 2-3 fue el parámetro que más influenció el modelo, a razón de que la mayoría de los pacientes en el modelo se encuentran en este rango que es el objetivo para la prevención de accidente cerebrovascular. Pero, la reducción del $10 \%$ del porcentaje de tiempo en rango de 3,1 y 3,9, TRT 1,5 y 1,9, TRT mayor a 4 y TRT menor de 1,5, ahorraría \$4 211957 037COP millones al año, que son parámetros modificables. El costo del tratamiento de las hemorragias intracraneales fue el segundo parámetro de mayor importancia en el modelo, debido al costo del tratamiento de las hemorragias moderadas y severas (la mayoría) que requieren manejo en unidad de cuidados intensivos y/o manejo quirúrgico en algunos casos y tiempo de estancia hospitalaria prolongados, por lo que es considerado el evento de mayor trascendencia, resaltado por varios autores ${ }^{15,21}$. Aunque el objetivo del modelo no contempló la variación en la prevalencia de la fibrilación auricular en Colombia, es posible modelar resultados en diferentes escenarios en los que se incluyan prevalencias diferentes al $3,6 \%$, por ejemplo $1-4 \%$, que es el comportamiento de otros países latinoamericanos.

El número de casos de este estudio fue inferior al reportado por Fanikos et al. ${ }^{26}$, que en su estudio de cohorte reportaron una tasa de eventos adversos entre 1 y $5 \%$, en tanto que en nuestro modelo fue de $0,78 \%$. Se considera que la mayor cantidad de eventos en el estudio de Fanikos et al. se debe a la inclusión de pacientes anticoagulados con warfarina por otras indicaciones y no exclusivamente por fibrilación auricular. De otro lado, la cohorte de Hilek 
et al. (de la cual se tomó la mayor cantidad de probabilidades), incluyó 13559 pacientes anticoagulados con warfarina por fibrilación auricular, el estudio más grande publicado y referente en el tema ${ }^{12}$. Diversos estudios han reportado el costo promedio del manejo de las hemorragias por warfarina; Fanikos et al. (EU), Abdelhafiz et al. (Inglaterra) y McBride et al. (Alemania) reportaron costos de $\$ 19$, £47 y $€ 15$ por paciente anticoagulado, mientras que en nuestro modelo el costo de los accidentes cerebrovasculares y sangrados en todos los pacientes anticoagulados fue de $\$ 43839$ COP (\$5 $598091 \times 0.0078311)$ o $\$ 15$ dólares estadounidenses aproximadamente ${ }^{26-28}$. La diferencia en costos se debe en parte al menor número de casos en este modelo y el menor costo de los servicios médicos en Colombia en comparación con los reportes de países desarrollados ${ }^{29}$.

Este modelo calculó el número y el costo de los eventos en pacientes anticoagulados con warfarina por fibrilación auricular; no se calculó el número total de casos de accidente cerebrovascular en Colombia, que es mayor a lo reportado en este estudio, ya que solo el $60 \%$ de los accidentes cerebrovasculares son tromboembólicos y de estos, solo el $15 \%$ son por fibrilación auricular; tampoco se calculó el costo total de todos los eventos adversos relacionados con el uso de warfarina por otras indicaciones - diferentes a fibrilación auricular. Una debilidad del modelo es la falta de datos para el caso Colombia, problema que todas las evaluaciones económicas enfrentan en el ámbito mundial ya que no existe toda la información para una población específica ${ }^{30}$. Consideramos que lograr TRT mayores al $60 \%$ en Colombia es un reto, por lo que la utilización de un porcentaje de TRT del $59 \%$ en el estudio, puede subestimar el cálculo de los eventos y el costo total de los mismos. Una de las fortalezas del modelo es la inclusión de los tiempos en rango terapéutico de la población, lo que permite modelar escenarios donde mayores o menores tasas pueden variar el costo para el sistema de salud. En este estudio se estimó que si se ajusta el tiempo en rango terapéutico en un $10 \%$ en la población que no se encuentra en rango terapéutico, se pueden ahorrar casi \$4 200 millones de pesos al año, cifra que puede ser importante para los tomadores de decisiones en Colombia.

Diversos autores han identificado las bajas tasas de tiempo en rango terapéutico como el principal problema en la terapia con warfarina, que se debe a múltiples factores intrínsecos y extrínsecos de los pacientes y de los sistemas de salud $^{31}$. Lader et al. afirman que al parecer pese a los esfuerzos de las estrategias para lograr más del $70 \%$ de tiempo en rango terapéutico, es imposible. Revisaron estudios en los que evaluaron diferentes estrategias como clínicas de anticoagulación, servicios de anticoagulación telefónicos y automonitorización de INR, en los que el TRT solo logró un $66,1 \%$ en el mejor de los casos; finalmente consideran que una estrategia múltiple podría lograr superar el $70 \%$ de $\mathrm{TRT}^{32}$. En Colombia, Laverde et al. y Taboada et al. describieron los resultados de una clínica de anticoagulación, en la que se reportaron tasas de eventos adversos y retrombosis y porcentaje de TRT en pacientes anticoagulados por todas las indicaciones; sin embargo no se comparó la clínica de anticoagulación con el manejo usual ${ }^{33,34}$. El cálculo del TRT en estudios clínicos se hizo por medio de modelos matemáticos complejos, por lo que para este modelo se empleó literatura internacional ${ }^{35}$; no obstante, debido a la heterogeneidad de resultados en el TRT, el análisis de sensibilidad ayuda a evaluar la variación de costos según diferentes escenarios de porcentaje de TRT.

El más reciente avance en anticoagulación son los nuevos anticoagulantes orales, que han mostrado resultados heterogéneos en la reducción de accidente cerebrovascular y eventos adversos en los ensayos clínicos pivote. La utilización de estos es reducida debido al acceso por el impacto presupuestario del costo para el sistema de salud y la precaución que se debe tener hasta que nuevos estudios post-autorización confirmen su perfil de seguridad y otras razones. Sin embargo, al parecer no existe reducción del accidente cerebrovascular, mortalidad y sangrados con dabigatrán $150 \mathrm{mg}$ cuando el TRT se encuentra por encima del $72,6 \%$ y no parece haber diferencias en los eventos según el TRT de warfarina con apixabán ${ }^{36,37}$, si bien ambos estudios pivote reportaron diferentes tasas de TRT.

\section{Conclusión}

La warfarina continuará siendo parte de las opciones en anticoagulación oral, pero se deben maximizar sus beneficios con estrategias múltiples que aseguren mejores tasas de TRT en la población. Este estudio estimó el número y el costo de los accidentes cerebrovasculares y de los eventos adversos de los pacientes anticoagulados con warfarina con fibrilación auricular no valvular. El costo médico y económico de los eventos adversos por warfarina es alto debido en mayor medida a hemorragias intracraneales y gastrointestinales, las cuales se pueden disminuir si se mejoran las tasas de tiempo en rango terapéutico de warfarina.

\section{Conflicto de intereses}

Ninguno.

\section{Agradecimientos}

A Red Cardiecol, Colciencias. CODIGO: 5020-53-731809

\section{Bibliografía}

1. Fuster V, Rydén LE, Cannom DS, Crijns HJ, Curtis AB, Ellenbogen KA, et al. 2011 ACCF/AHA/HRS Focused Updates Incorporated Into the ACC/AHA/ESC 2006 Guidelines for the Management of Patients With Atrial Fibrillation. J Am Coll Cardiol. 2011;57:e101-98

2. Go AS, Hylek EM, Phillips KA, Chang Y, Henault LE, Selby JV, et al. Prevalence of Diagnosed Atrial Fibrillation in Adults. JAMA. 2001;285:2370.

3. Kistler JFK. Carotid Endarterectomy Revisited. N Engl J Med. 2000.

4. Ghate SR, Biskupiak J, Ye X, Kwong WJ, Brixner DI. All-Cause and Bleeding-Related Health Care Costs in Warfarin-Treated Patients with Atrial Fibrillation. J Manag Care Pharm. 2011;17:672-84.

5. Gurwitz JH, Field TS, Harrold LR, Rothschild J, Debellis K, Seger AC, et al. Incidence and Preventability of in the Ambulatory Setting. J Am Med Assoc. 2003;289:1107-16.

6. Johnsen SP, Sørensen HT, Mellemkjoer L, Blot WJ, Nielsen GL, McLaughlin JK, et al. Hospitalisation for Upper Gastrointestinal 
Bleeding Associated with Use of Oral Anticoagulants. Thromb Haemost. 2001;86:563-8.

7. Pirmohamed M, James S, Meakin S, Green C, Scott AK, Walley TJ, et al. Adverse drug reactions as cause of admission to hospital: prospective analysis of 18820 patients. BMJ. 2004;329:15-9.

8. Amorosi SL, Armstrong S, Da Deppo L, Garfield S, Stein K. The budget impact of left atrial appendage closure compared with adjusted-dose warfarin and dabigatran etexilate for stroke prevention in atrial fibrillation. Europace. 2014;16:1131-6.

9. Un TPOR. Guía de Práctica Clínica para el diagnóstico, tratamiento y rehabilitación del episodio agudo del Ataque Cerebrovascular Isquémico en población mayor de 18 años. 2015.

10. Mercaldi CJ, Ciarametaro M, Hahn B, Chalissery G, Reynolds MW, Sander SD, et al. Cost efficiency of anticoagulation with warfarin to prevent stroke in medicare beneficiaries with nonvalvular atrial fibrillation. Stroke. 2011;42:112-8.

11. Wang C, Yang Z, Wang C, Wang Y, Zhao X, Liu L, et al. Significant underuse of warfarin in patients with nonvalvular atrial fibrillation: Results from the China National Stroke Registry. J Stroke Cerebrovasc Dis. 2014;23:1157-63.

12. Hylek H, Go ASYC. Effect of intensity of oral anticoagulation on stroke severity and mortality in atrial fibrillation. nejm. 2010;2010:1463-76.

13. Capodanno D, Capranzano P, Giacchi G, Calvi V, Tamburino C. Novel oral anticoagulants versus warfarin in non-valvular atrial fibrillation: A meta-analysis of 50,578 patients. Int J Cardiol. 2013;167:1237-41.

14. Corey SM, Sonia MG, Shimony A, Kristian BFi, Eisenberg MJ. Meta-Analysis of Efficacy and Safety of New Oral Anticoagulants(dabigatran, rivaroxaban, apixavan) Versus Warfarin in Patients With Atrial Fibrillation. Am J Cardiol. 2012;110:453-60.

15. Kam JK, Chen Z, Liew D, Yan B. Does warfarin-related intracerebral haemorrhage lead to higher costs of management? Clin Neurol Neurosurg. 2014;126:38-42.

16. Lauer A, Pfeilschifter W, Schaffer CB, Lo EH, Foerch C. Intracerebral haemorrhage associated with antithrombotic treatment: Translational insights from experimental studies. Lancet Neurol. 2013;12:394-405.

17. Sullivan SD, Mauskopf JA, Augustovski F, Jaime Caro J, Lee KM, Minchin M, et al. Budget impact analysis - Principles of good practice: Report of the ISPOR 2012 budget impact analysis good practice II task force. Value Heal. 2014;17:5-14.

18. Mauskopf Ja, Sullivan SD, Annemans L, Caro J, Mullins CD, Nuijten $M$, et al. Principles of good practice for budget impact analysis: report of the ISPOR Task Force on good research practices-budget impact analysis. Value Heal. 2007;10:336-47.

19. Rosselli D, Rodríguez AJ, García ÁA, Rueda JD. Prevalencia de fibrilación auricular en un hospital universitario colombiano. Rev Colomb Cardiol. 2013;20:383-5.

20. Mohammed MA, Marshall T, Nirantharakumar K, Stevens A, Fitzmaurice D. Patterns of Warfarin Use in Subgroups of Patients with Atrial Fibrillation: A Cross-Sectional Analysis of 430 General Practices in the United Kingdom. PLoS One. 2013;8:1-8.
21. Rosand J, Eckman MH, Knudsen KA, Singer DE, Greenberg SM. The Effect of Warfarin and Intensity of Anticoagulation on Outcome of Intracerebral Hemorrhage. Arch Intern Med. 2004; $164: 880$.

22. Chen W-C, Chen Y-H, Hsu P-I, Tsay F-W, Chan H-H, Cheng J-S, et al. Gastrointestinal Hemorrhage in Warfarin Anticoagulated Patients: Incidence, Risk Factor. Management, and Outcome. Biomed Res Int. 2014;2014:1-7.

23. Paul Leigh J, White RH. An economic model of adverse events and costs for oral anticoagulants used for atrial fibrillation. Curr Med Res Opin. 2007;23:2071-81.

24. DANE. Colombia. Proyecciones de poblaciones municipales.

25. Coppens $M$. New oral anticoagulants versus vitamin $\mathrm{K}$ antagonists in countries with good INR control. Neth J Med. 2013;71:168-9.

26. Fanikos J, Grasso-Correnti N, Shah R, Kucher N, Goldhaber SZ. Major bleeding complications in a specialized anticoagulation service. Am J Cardiol. 2005;96:595-8.

27. Abdelhafiz AH, Wheeldon NM. Use of resources and cost implications of stroke prophylaxis with warfarin for patients with nonvalvular atrial fibrillation. Am J Geriatr Pharmacother. 2003;1:53-60.

28. Mcbride D, Mattenklotz AM, Willich SN, Brüggenjürgen B. The Costs of Care in Atrial Fibrillation and the Effect of Treatment Modalities in Germany. Value Heal. 2009;12:293-301.

29. Puente MDe. Health tourism sector?: The colombian case. Rev Econ del Caribe. 2015;2106:129-61.

30. Weinstein MC, O’Brien B, Hornberger J, Jackson J, Johannesson $\mathrm{M}$, McCabe $\mathrm{C}$, et al. Principles of good practice for decision analytic modeling in health-care evaluation: Report of the ISPOR task force on good research practices - Modeling studies. Value Heal. 2003;6:9-17.

31. Beyth RJ, Quinn L, Landefeld CS. A multicomponent intervention to prevent major bleeding complications in older patients receiving warfarin. Ann Intern Med. 2000;133:687-95.

32. Lader E, Martin N, Cohen G, Meyer M, Reiter P, Dimova A, et al. Warfarin therapeutic monitoring: Is $70 \%$ time in the therapeutic range the best we can do? J Clin Pharm Ther. 2012;37:375-7.

33. Laverde LP, Gómez SE, Montenegro AC, Lineros A, Wills B, Buitrago AF. Experiencia de una clínica de anticoagulación. Rev Colomb Cardiol. 2015;22:224-30.

34. Taboada LB, Silva LE, Montenegro AC. Beneficios de la clínica de anticoagulación. Acta Médica Colomb. 2013;38:239-43.

35. Rosendaal FR, Cannegieter SC, Van der Meer FJM, Briet E. A method to determine the optimal intensity of oral anticoagulant therapy. Thromb Haemost. 1993;69:236-9.

36. Wallentin L, Yusuf S, Ezekowitz MD, Alings M, Flather M, Franzosi MG, et al. Efficacy and safety of dabigatran compared with warfarin at different levels of international normalised ratio control for stroke prevention in atrial fibrillation: An analysis of the RE-LY trial. Lancet. 2010;376:975-83.

37. Wallentin L, Lopes RD, Hanna M, Thomas L, Hellkamp A, Nepal $S$, et al. Efficacy and safety of apixaban compared with warfarin at different levels of predicted international normalized ratio control for Stroke prevention in atrial fibrillation. Circulation. 2013;127:2166-76. 